\title{
Treatment of persistent bacterial vaginosis and risk for spontaneous preterm birth
}

\author{
Yair Blumenfeld ${ }^{1}$, Ivana Maric ${ }^{1}$, David Stevenson ${ }^{2}$, Ronald Gibbs ${ }^{1}$, and Gary Shaw ${ }^{3}$ \\ ${ }^{1}$ Stanford University School of Medicine \\ ${ }^{2}$ Lucile Salter Packard Children's Hospital at Stanford \\ ${ }^{3}$ Stanford University
}

April 8, 2021

\begin{abstract}
Objective: To determine the association between treatment of persistent BV in pregnancy and risk for spontaneous preterm birth (sPTB). Design: The retrospective data from IBM@ MarketScanß Commercial Database was analyzed. Setting: United States outpatient data. Population or Sample: Women aged $12-55$ years with a singleton gestation. Methods: Women were linked to an outpatient medications database and medications taken during the pregnancy were analyzed. Treatment of BV in pregnancy was defined as a diagnosis of BV and treatment with Metronidazole and/or Clindamycin, and persistent treatment of $\mathrm{BV}$ was defined as $\mathrm{BV}$ in more than 1 trimester or $\mathrm{BV}$ requiring more than 1 antibiotic prescription. Odds ratios were calculated comparing SPTB in those with BV and persistent BV to women without BV in pregnancy. Survival analysis using Kaplan-Meier curves for the gestational age at delivery was also performed. Main outcome measures: sPTB Results: Among a cohort of 2,538,606 women, 216,611 had an associated ICD-9 or ICD-10 code for diagnosis of BV alone, and 63,817 had BV and were treated with either metronidazole and/or clindamycin. The sPTB rate among women treated with BV was $7.5 \%$ compared with $5.7 \%$ for women without BV who did not receive antibiotics. Relative to those without BV in pregnancy, odds ratios for sPTB were highest in those treated for BV in both the first and second trimester (1.66 [95\% CI 1.52, 1.81]) or those with 3 or more prescriptions in pregnancy $(1.48$ [95\% CI 1.35, 1.63]. Conclusions: Treatment of persistent BV is associated with increased sPTB risk.
\end{abstract}

Treatment of persistent bacterial vaginosis and risk for spontaneous preterm birth

Yair J. Blumenfeld, $\mathrm{MD}^{1}$, Ivana Marić, $\mathrm{PhD}^{2}$, David K. Stevenson, $\mathrm{MD}^{2}$, Ronald S. Gibbs, $\mathrm{MD}^{1}$, Gary M Shaw, $\mathrm{DrPH}^{2}$

${ }^{1}$ Department of Obstetrics \& Gynecology, Stanford University School of Medicine, 94305

${ }^{2}$ Department of Pediatrics, Stanford University School of Medicine, March of Dimes Prematurity Research Center, Stanford, California, 94305

Corresponding author:

Yair J. Blumenfeld, MD

Associate Professor

Department of Obstetrics \& Gynecology

Stanford University School of Medicine

300 Pasteur Drive, Room H304B 
Stanford, California, 94305

yairb@stanford.edu

650-269-4665

Running Title: Treatment of persistent bacterial vaginosis in pregnancy is associated with a higher risk for spontaneous preterm birth.

Abstract:

Objective:

To determine the association between treatment of persistent BV in pregnancy and risk for spontaneous preterm birth (sPTB).

Design:

The retrospective data from IBM@ MarketScan@ Commercial Database was analyzed.

Setting:

United States outpatient data.

Population or Sample:

Women aged $12-55$ years with a singleton gestation.

Methods:

Women were linked to an outpatient medications database and medications taken during the pregnancy were analyzed. Treatment of BV in pregnancy was defined as a diagnosis of BV and treatment with Metronidazole and/or Clindamycin, and persistent treatment of BV was defined as BV in more than 1 trimester or BV requiring more than 1 antibiotic prescription. Odds ratios were calculated comparing sPTB in those with BV and persistent BV to women without BV in pregnancy. Survival analysis using Kaplan-Meier curves for the gestational age at delivery was also performed.

Main outcome measures:

sPTB

Results:

Among a cohort of 2,538,606 women, 216,611 had an associated ICD-9 or ICD-10 code for diagnosis of BV alone, and 63,817 had BV and were treated with either metronidazole and/or clindamycin. The sPTB rate among women treated with BV was $7.5 \%$ compared with $5.7 \%$ for women without BV who did not receive antibiotics. Relative to those without BV in pregnancy, odds ratios for sPTB were highest in those treated for BV in both the first and second trimester $(1.66$ [95\% CI 1.52, 1.81]) or those with 3 or more prescriptions in pregnancy (1.48 [95\% CI 1.35, 1.63].

Conclusions:

Treatment of persistent BV is associated with increased sPTB risk.

Keywords: Bacterial Vaginosis, spontaneous preterm birth, recurrent

Introduction:

Bacterial vaginosis (BV) results from a change in the normal microbial environment of the vagina, with a lack of hydrogen peroxide-producing lactobacilli and an overgrowth of facultative anaerobic organisms including Gardnerella vaginalis, Bacteroides species, Mycoplasma hominis, Peptostreptococcus species, and other species. ${ }^{1}$ Approximately $20-50 \%$ of reproductive age women will experience an episode of BV, and 6$19 \%$ will have an episode of BV during pregnancy, ${ }^{2,3}$ An underlying infectious or inflammatory etiology is one 
of the suspected leading contributors to spontaneous preterm birth (sPTB), and the association between BV and sPTB has been previously described in multiple studies. ${ }^{4}$ Despite this known association, screening and treatment of BV in pregnancy as a means of reducing SPTB remains controversial. A recent US Preventative Task Force addressing screening for BV to prevent preterm delivery "recommended against screening for bacterial vaginosis in pregnant persons not at increased risk for preterm delivery." 5

Although for most women an episode of BV will resolve without any difficulty or long term sequalae, persistent BV, defined as BV that recurs or fails to improve after an initial antibiotic course, complicates $20-75 \%$ of all cases. ${ }^{6}$ Persistent BV has been linked with a variety of adverse effects including discomfort and pain, negative emotional, sexual, and social effects. ${ }^{7}$ While the association between first trimester BV with sPTB has been previously described, there is a paucity of data about the association between treatment of persistent BV during pregnancy and sPTB. Our aim was to determine whether treatment of persistent BV is associated with a higher risk for sPTB.

Methods:

Determining Pregnancy Cohort

IBM@ MarketScan@ Commercial Database that includes health insurance claims data from across the US from 2007 to 2016 were used. A cohort of women aged 12-55 years with singleton pregnancies was identified from the IBM MarketScan Commercial Database inpatient admissions by using International Classification of Diseases, 9th Revision, Clinical Modification (ICD-9) diagnosis codes (640-649 (Complications mainly related to pregnancy), 650, 652-659 (Normal delivery, and other indications for care in pregnancy, labor, and delivery), 660-669 (Complications occurring mainly in the course of labor and delivery), 670-677 (Complications of the puerperium), 678-679 (Other maternal and fetal complications), V27.0, V27.1, V27.9 (Outcome of delivery); only codes ending in ${ }^{* * *} . * 1$ and $* * * . * 2$ identifying delivery were used), ICD-10 codes (O80O83 (Single delivery), Z37.0, Z37.1, Z37.9 (Outcome of delivery), O60.1*X0, O60.1*X1 (Preterm labor with preterm delivery), O42.1 except O42.111, O42.1 except O42.111, O42.9 except O42.911 (Premature rupture of membranes)(Marić et al. 2019)), diagnosis-group related (DRG) codes (765-766 (Cesarean section with/without complication or comorbidity), 767 (Vaginal delivery with sterilization and/or D\&C), 768 (Vaginal delivery with O.R. procedure except sterilization and/or D\&C), 774-775 (vaginal delivery with/without complications)) and procedure codes (72.** (Forceps, vacuum, and breech delivery), 73.22, 73.59, 73.6 (Other procedures inducing or assisting delivery), 74.0-74.2, 74.4, 74.99 (Cesarean section and removal of fetus)) as previously described in Marić et. al. ${ }^{8}$ Given the differences in sPTB risk between singleton and multiple gestations, deliveries with codes for multi-fetal pregnancies (ICD-9 codes 651, V27.2-V27.7, V-91; ICD-10 codes O30) were excluded. To avoid dependencies for women with multiple pregnancies, only the first pregnancy recorded in the IBM MarketScan Commercial Database was included. We excluded molar and ectopic pregnancies (ICD-9 codes 630 and 633; ICD-10 codes O00 and O01; DRG code 777), spontaneous and induced abortions (ICD-9 codes 632, 634-637; ICD-10 codes O03), complications following abortion, ectopic or molar pregnancies (ICD-9 code 639; ICD-10 codes O04 and O08), and abortions with/without D\&C (DRG codes 770 and 779$)$.

Gestational age (GA) at delivery was estimated by using the algorithm described in Marić et. al. ${ }^{8}$ Briefly, the algorithm is based on codes for the number of completed weeks of gestation (ICD-9 codes 765.2, 766.21, 766.22; ICD-10 codes Z3A) and codes for preterm labor with preterm delivery (ICD- 9644.21; ICD-10 codes O60.1*X0), identified directly from women's records when present or, otherwise, from infants' records. To obtain GAs from infant records, single liveborn infants were identified (ICD-9 codes V30 and V39; ICD-10 codes Z38.0-Z38.2) and linked to their mothers. Women without a GA code either in their own or their infant record, were assigned a GA of: 1) 34 weeks if they were identified to have sPTB, as described below, or 2) 39 weeks, otherwise.

\section{Identifying spontaneous PTB risk}

For women without a GA code in their records, the distinction of sPTB was made by codes for preterm labor with preterm delivery (ICD-9 code 644.21; ICD-10 codes O60.1*X0, O42.012). For women with a GA 
code, sPTB was identified by a GA code $<37$ weeks accompanied by a code for the following diagnoses:

1. premature rupture of membranes (ICD-9 codes 658.1; ICD-10 codes O42.012, O42.013, O42.019, O42.112, O42.113, O42.119, O42.9 except O42.911);

2. early or threatened preterm labor (ICD-9 codes 644.*; ICD-10 codes O60.1*X0, O60.0).

\section{Continuous Enrollment Requirement}

Women who were continuously enrolled for at least 9 months prior to admission for delivery were included.

Identifying Patients with Bacterial Vaginosis and Treatment

Within the pregnancy cohort two groups of women were identified and compared. The first group consisted of women identified as having BV by diagnostic codes (ICD-9 codes 616.10, 616.11; ICD-10 codes N76.0, N76.1, N76.2, N76.3, N77.1) occurring at any time during pregnancy. Women in this group were treated with Metronidazole and/or Clindamycin, as indicated by at least one prescription record. Three time windows were considered for the treatment: 1) duration of the pregnancy; 2) first trimester, or 3) second trimester. The referent group consisted of women with no BV and no treatment with either of the two medications during pregnancy. Odds ratios for the associations with sPTB were calculated using logistic regression along with $95 \%$ confidence intervals.

To examine the association between treatment of persistent BV and sPTB we performed an additional analysis where the women with BV and medication prescription filled during pregnancy were stratified based on the number of prescriptions that were filled. To further examine the differences between women with BV and treatments vs. referent group, we performed survival analysis and obtained Kaplan-Meier curves for the time of delivery for the two groups of women.

This project was deemed exempt by the Stanford Institutional Review Board for Human Subjects.

Results:

Of 15,665,160 patients in the IBM MarketScan Commercial Database inpatient admissions between 2007 and 2016, we extracted pregnancies with a singleton gestation and continuous coverage during pregnancy and excluded those with molar pregnancies, ectopic pregnancies and abortions (figure 1). After excluding those in which the gestational age at delivery could not be verified a total of 2,538,606 women were included in the final analysis. A total of 216,611 (8.5\%) were identified as having a ICD-9 or ICD-10 code for BV, and of those, 63,817 women $(2.5 \%)$ had both a diagnosis of BV and filled a prescription for either metronidazole and/or clindamycin (supplemental figure). Among the 63,817 women $72.0 \%$ filled only one prescriptions during pregnancy, $19.2 \%$ filled 2 prescriptions, and $8.8 \%$ filled 3 or more prescriptions (figure 2). $56 \%$ of prescriptions were filled before 20 weeks' gestation with a peak at 13 weeks of gestation. A second peak was found around 33 weeks' gestation (figure 3).

Spontaneous PTB occurred in 150,118 (5.9\%) women in the cohort (supplemental table). sPTB among women treated for BV was $7.5 \%$ while those without a diagnosis of BV had a frequency of sPTB of $5.7 \%$. Compared with women who were not diagnosed with BV, the odds ratio for SPTB in those treated for BV in the first trimester was 1.42 [95\% CI: 1.36,1.48], for those treated in the second trimester was 1.47 [95\% CI: $1.41,1.53]$ and for those treated in both the first and second trimester was 1.66 [95\% CI: 1.52, 1.81] (Table 1).

Similarly, there was an increased risk for sPTB in women treated for BV in more than 1 trimester compared to women without BV in pregnancy. Specifically, the OR for those with 1 prescription was 1.30 [95\% CI: 1.26, 1.35] while for those with 2 prescriptions 1.35 [95\% CI: 1.26, 1.44] and those with 3 or more prescriptions 1.48 [95\% CI: 1.35, 1.63] (Table 2). A Kaplan Meyer survival curve for sPTB stratified by antibiotic treatment (metronidazole and/or clindamycin) illustrating the difference in survival for two groups is shown in Figure 4 and, specifically for the period from 30 to 39 weeks in Figure 5. Most of the sPTB difference between those with BV and without BV was seen in the late preterm period, between 34 and 37 weeks' gestation. 


\section{Discussion:}

In a large US cohort of pregnant women we observed that treatment of persistent BV, defined as either BV occurring in more than 1 trimester or requiring more than 1 prescription, was associated with an increased risk for $\mathrm{sPTB}$ relative to not having BV. These observed risks were higher than the risk of sPTB observed for women with a single treatment of BV. Those filling at least 3 prescriptions and those with therapy in both the first and second trimester had an approximately $50 \%$ increased risk for sPTB. It is unclear whether persistent BV directly led to intra-amniotic infection and $\mathrm{SPTB}$, or whether BV may be a marker of different risk factors for sPTB that we could not account for in our dataset.

BV can be diagnosed in several ways. The reference standard for BV diagnosis is the Nugent score, although it's use is generally limited to research settings. ${ }^{1}$ Nugent scoring assigns a value to different bacterial morphotypes seen on Gram stain of vaginal secretions with a score $>7$ interpreted as bacterial vaginosis. More often BV is diagnosed by the presence of 3 of 4 Amsel criteria, which has been shown to correlate with the Nugent scoring. The incidence of BV in pregnancy likely depends on the population studied (high vs. low risk for BV) and the method of diagnosis. In a prospective study of 148 women, 65 women (43.9\%) were diagnosed in the first trimester using a Nugent score $>7,32$ women $(21.6 \%)$ were diagnosed in the second trimester, and 28 women $(18.9 \%)$ were diagnosed in the third trimester. ${ }^{9}$ In that cohort only $48 \%$ were negative in all trimesters and $9.4 \%$ were positive throughout pregnancy. It's important to note however that women were screened irrespective of symptoms, and most women were young (mean $=23)$, unmarried $(76 \%)$, African American (85\%) and women of low income. A much lower BV incidence was found in a multicenter double blind randomized BV therapy trial from France, for example, in which only 5630 of 84530 women $(6.6 \%)$ had BV based on a diagnosis of a Nugent score $>7 .^{10}$ The 63,817 women with BV based on ICD-9 and ICD-10 coding along with a prescription for BV therapy in our analysis represented $2.7 \%$ of the overall pregnancy cohort, suggesting a relatively low prevalence group and one that was possibly screened based on symptoms, rather than universal screening paradigms. It is also possible that our relatively low rate resulted from the fact that some women were prescribed metronidazole and/or clindamycin for BV but were not coded as such. In fact, it is important to note that 134,737 women received a prescription for metronidazole and/or clindamycin without a code specific for BV. When we analyzed the association between such treatment and sPTB, we found an increased risk, although not as high as in those with both an ICD-9/10 code and treatment (Table S1 to S5, Figure S1 to S3).

The association between BV and sPTB has been known for over 2 decades. In a prospective study of 2929 pregnancies conducted by the Maternal Fetal Medicine Units Network between 1992 and 1994 the presence of BV was associated with a 2.7 relative risk of sPTB before 32 weeks gestation. ${ }^{4}$ Subsequent cohort studies have confirmed this association, and there are several demographic risk factors that are similar for BV and sPTB. The precise mechanism in which BV leads to sPTB remains unknown although an increased risk of ascending infection has been hypothesized. A link between first trimester BV and short cervix, a risk factor for sPTB, has also been found; unfortunately, we were not able to assess cervical length in our study. ${ }^{11}$ Additionally, whether persistent BV represents a higher microbial load thereby increasing the risk of intraamniotic infection and inflammation remains to be seen. It is also worth noting that in our cohort, based on our Kaplan-Meier graphs, the greatest risk was seen at late preterm gestational ages (34-37 weeks). This is contrary to some of the earlier reports associating BV with preterm birth before 35 weeks. ${ }^{4}$

While the association between BV and sPTB has been previously described, studies including several prospective clinical trials have failed to demonstrate that treating $\mathrm{BV}$ in pregnancy alone reduces the risk for sPTB. ${ }^{10,12-14}$ Reasons for this remain unclear, although possible explanations could be poor compliance, inappropriate diagnosis, inappropriate treatment, or treatment after the beginning of an ascending infection or short cervix. In addition, more recent studies suggest that not all BV cases are caused by similar bacteria, particularly across different population subgroups. ${ }^{15}$ Unfortunately we were unable to determine the contribution of maternal race/ethnicity on sPTB risk in our cohort. Moreover, previous studies suggest that antibiotic therapy alone may increase the risk of sPTB. ${ }^{16}$ While antibiotics alone in those screened for BV have not been shown to reduce sPTB incidence, identifying high risk cohorts has potential important clinical 
implications. For example, transvaginal ultrasound cervical length surveillance is routinely performed in other high risk cohorts and adjunct therapy with vaginal progesterone therapy and/or cerclage has been shown to lower sPTB in those with a decreasing cervical length. How to incorporate such interventions for women with persistent BV or lasting beyond 2 trimesters remains to be seen, and future studies are warranted to further delineate the precise pathophysiologic mechanism between BV and sPTB so that an appropriate intervention can be identified.

Our study has several notable strengths. First, we were able to analyze a robust cohort of over $2.5 \mathrm{M}$ pregnancies, from diverse geographical areas in the United States. We defined treatment of BV via the presence of BV by both diagnostic codes and antibiotic treatment using two of the most common and recommended medications. ${ }^{1}$ While clindamycin can also be used for urinary tract infections (which we excluded in our analysis), metronidazole is rarely used in pregnancy for indications other than BV. A less common use for metronidazole is trichomonas infections, but we did exclude those codes in our dataset and the incidence of trichomonas in pregnancy is much lower than BV. The dataset allowed us to assess diagnoses and therapy over the entire course of the pregnancy, and to link it with clinical outcome and with medication claims. Specifically, the dataset is unique in that we were able to delineate sPTB using a previously validated analytical approach, excluding other indicated PTB codes such as preeclampsia.

On the other hand, our study is not without limitations. Due to the lack of certain data collected in this administrative dataset we were unable to account for baseline demographic information including maternal race, ethnicity, BMI and smoking status. This is particularly important as previous studies have shown differences in underlying vaginal microbial signatures among different races with important implications for sPTB risk. ${ }^{17,18}$ It is also unclear whether women diagnosed with BV were symptomatic, and whether any additional over the counter interventions were employed. This is an important factor to consider since women who are screened rather than presenting with symptoms may be deemed to be at higher risk by their providers which may lead to selection bias. The method of BV diagnosis in the cohort is also not available, and it is possible that women were empirically treated and coded as having BV without an actual clinical diagnosis. Treatment adherence/non-compliance is also an issue which may bias our results and because we used antibiotic use to support our diagnosis of BV we were unable to reliably determine the effect of antibiotic therapy on sPTB risk. We were also unable to delineate the route of administration, and whether an additional antibiotic prescription was prescribed because of difficulty with administration route vs treatment failure. Additionally, the dataset is a private insurance outpatient dataset and selects for a certain demographic and therefore may not be generalizable to the US population as whole. Finally, the increased risks observed between treatment of persistent BV and sPTB were relative to women not having $\mathrm{BV}$. Thus, we cannot disentangle whether occurrence of BV or its treatment was the underlying driver of the observed increased risk.

\section{Conclusion:}

In conclusion, our data indicate that persistent BV infection in pregnancy is associated with increased sPTB risk. Given the rates of persistent BV our data highlight the importance of clinical follow-up after the initial diagnosis and therapy of BV in pregnancy. Future studies are warranted to elucidate the precise mechanism in which persistent BV may lead to SPTB and also to further clarify whether therapy in such cases is an appropriate measure to reduce preterm birth.

Data Availability Statement:

IBM MarketScan Research Databases are available to purchase by Federal, nonprofit, academic, pharmaceutical, and other researchers. Use of the data is contingent on completing

a data use agreement and purchasing the data needed to support the study. More information about licensing the IBM MarketScan Research Databases is available at:

https://www.ibm.com/products/marketscan-research-databases

Disclosure of Interests: The authors have not financial interests to disclose related to this work 
Authors contribution: YJB, IM and GMS were involved in the conception and planning of the study, data analysis, and manuscript development. DKS was involved in planning the study, data analysis and manuscript development. RSG was involved in data analysis and manuscript development.

Ethics approval: This project was deemed exempt by the Stanford Institutional Review Board for Human Subjects.

Funding: This work was supported by the March of Dimes Prematurity Research Center at Stanford University and the Maternal and Child Health Research Institute, Stanford University.

References

1. Committee on Practice B-G. Vaginitis in Nonpregnant Patients: ACOG Practice Bulletin, Number 215. Obstet Gynecol.2020;135(1):e1-e17.

2. Bruggman A, Ortiz-Hartman K. Community $\&$ family health issues : an encyclopedia of trends, conditions $\&$ treatments. First edition . ed. Ipswich, Massachusetts

Amenia, New York: Salem Press ;

Grey House Publishing; 2017.

3. Cohen CR, Wierzbicki MR, French AL, et al. Randomized Trial of Lactin-V to Prevent Recurrence of Bacterial Vaginosis. N Engl J Med. 2020;382(20):1906-1915.

4. Goldenberg RL, Iams JD, Mercer BM, et al. The preterm prediction study: the value of new vs standard risk factors in predicting early and all spontaneous preterm births. NICHD MFMU Network. Am J Public Health. 1998;88(2):233-238.

5. Force USPST, Owens DK, Davidson KW, et al. Screening for Bacterial Vaginosis in Pregnant Persons to Prevent Preterm Delivery: US Preventive Services Task Force Recommendation Statement. JAMA.2020;323(13):1286-1292.

6. Sena AC, Lee JY, Schwebke J, et al. A Silent Epidemic: The Prevalence, Incidence and Persistence of Mycoplasma genitalium Among Young, Asymptomatic High-Risk Women in the United States. Clin Infect Dis. 2018;67(1):73-79.

7. Nyirjesy P. Management of persistent vaginitis. Obstet Gynecol. 2014;124(6):1135-1146.

8. Maric I, Winn VD, Borisenko E, et al. Data-driven queries between medications and spontaneous preterm birth among 2.5 million pregnancies.Birth Defects Res. 2019;111(16):1145-1153.

9. Waters TP, Denney JM, Mathew L, Goldenberg RL, Culhane JF. Longitudinal trajectory of bacterial vaginosis during pregnancy.Am J Obstet Gynecol. 2008;199(4):431 e431-435.

10. Subtil D, Brabant G, Tilloy E, et al. Early clindamycin for bacterial vaginosis in pregnancy (PREMEVA): a multicentre, double-blind, randomised controlled trial. Lancet. 2018;392(10160):2171-2179.

11. Donders GG, Van Calsteren C, Bellen G, et al. Association between abnormal vaginal flora and cervical length as risk factors for preterm birth. Ultrasound Obstet Gynecol. 2010.

12. Bellad MB, Hoffman MK, Mallapur AA, et al. Clindamycin to reduce preterm birth in a low resource setting: a randomised placebo-controlled clinical trial. BJOG. 2018;125(12):1601-1609.

13. Anderson B, Zhao Y, Andrews WW, et al. Effect of antibiotic exposure on Nugent score among pregnant women with and without bacterial vaginosis. Obstet Gynecol. 2011;117(4):844-849.

14. Haahr T, Ersboll AS, Karlsen MA, et al. Treatment of bacterial vaginosis in pregnancy in order to reduce the risk of spontaneous preterm delivery - a clinical recommendation. Acta Obstet Gynecol Scand. 2016;95(8):850-860. 
15. Foxman B, Wen A, Srinivasan U, et al. Mycoplasma, bacterial vaginosis-associated bacteria BVAB3, race, and risk of preterm birth in a high-risk cohort. Am J Obstet Gynecol. 2014;210(3):226 e221-227.

16. Klebanoff MA, Carey JC, Hauth JC, et al. Failure of metronidazole to prevent preterm delivery among pregnant women with asymptomatic Trichomonas vaginalis infection. N Engl J Med.2001;345(7):487-493.

17. Callahan BJ, DiGiulio DB, Goltsman DSA, et al. Replication and refinement of a vaginal microbial signature of preterm birth in two racially distinct cohorts of US women. Proc Natl Acad Sci $U S$ A.2017;114(37):9966-9971.

18. Meis PJ, Goldenberg RL, Mercer BM, et al. Preterm prediction study: is socioeconomic status a risk factor for bacterial vaginosis in Black or in White women? Am J Perinatol. 2000;17(1):41-45.

Legends of the figures and tables:

Figure 1. Pregnancy cohort flow chart.

Figure 2. Histogram of number of women with BV that filled 1, 2, or 3 and more prescriptions for metronidazole and/or clindamycin during pregnancy.

Figure 3. Histogram of number of women who filled a prescription per each week of pregnancy.

Table 1. Odds ratios for spontaneous PTB according to treatment for bacterial vaginosis by trimester of pregnancy. For each exposed group the comparison is performed against the group of patients without BV and without exposure to medication as shown in the last row.

Table 2. Odds ratios for spontaneous preterm births by number of prescriptions during pregnancy. For each group the comparison is performed against the group of patients without BV and without exposure to medication as shown in the last row.

Figure 4. Survival curves for spontaneous preterm birth stratified by Metronidazole and/or Clindamycin treatment.

Figure 5. Survival curves for spontaneous preterm birth stratified by Metronidazole and/or Clindamycin treatment for 30 to 39 gestational weeks. Dashed lines show confidence intervals.

Figure 1. Pregnancy cohort flow chart.

\section{Hosted file}

image1.emf available at https://authorea.com/users/406464/articles/517114-treatment-ofpersistent-bacterial-vaginosis-and-risk-for-spontaneous-preterm-birth

Figure 2. Histogram of number of women with BV that filled 1, 2, or 3 and more prescriptions for metronidazole and/or clindamycin during pregnancy.

\section{Hosted file}

image2.emf available at https://authorea.com/users/406464/articles/517114-treatment-ofpersistent-bacterial-vaginosis-and-risk-for-spontaneous-preterm-birth

Figure 3. Histogram of number of women who filled a prescription per each week of pregnancy.

\section{Hosted file}

image3.emf available at https://authorea.com/users/406464/articles/517114-treatment-ofpersistent-bacterial-vaginosis-and-risk-for-spontaneous-preterm-birth

Table 1. Odds ratios for spontaneous PTB according to treatment for bacterial vaginosis by trimester of pregnancy. For each exposed group the comparison is performed against the group of patients without BV and without exposure to medication as shown in the last row. 


\begin{tabular}{lllll}
\hline & $\#$ PTB n(\%) & Term & Total & OR [95\% CI] \\
\hline In pregnancy & $4,787(7.5)$ & 59,030 & 63,817 & $1.33[1.29,1.37]$ \\
$\mathbf{1}^{\text {st }}$ trimester & $2142(8.0)$ & 24,736 & 26,878 & $1.42[1.36,1.48]$ \\
$\mathbf{2}^{\text {nd }}$ trimester & $2630(8.2)$ & 29,286 & 31,916 & $1.47[1.41,1.53]$ \\
$\mathbf{1}^{\text {st }}$ and 2 \\
No BV \& \\
no medication & $553(9.2)$ & 5,442 & 5,995 & $1.66[1.52,1.81]$ \\
\hline
\end{tabular}

Table 2. Odds ratios for spontaneous preterm births by number of prescriptions during pregnancy. For each group the comparison is performed against the group of patients without BV and without exposure to medication as shown in the last row.

\begin{tabular}{lllll}
\hline \# of prescriptions & \# PTB n(\%) & Term & Total & OR [95\% CI] \\
\hline $\mathbf{1}$ & $3388(7.3)$ & 42,565 & 45,953 & $1.30[1.26,1.35]$ \\
$\mathbf{2}$ & $935(7.6)$ & 11,347 & 12,282 & $1.35[1.261 .44]$ \\
$\mathbf{3} \mathbf{3}$ & $464(8.3)$ & 5118 & 5582 & $1.48[1.35,1.63]$ \\
No BV \& & & & & \\
no medication & $125,815(5.7)$ & $2,061,443$ & $2,187,258$ & \\
\hline
\end{tabular}

Figure 4. Survival curves for spontaneous preterm birth stratified by Metronidazole and/or Clindamycin treatment.

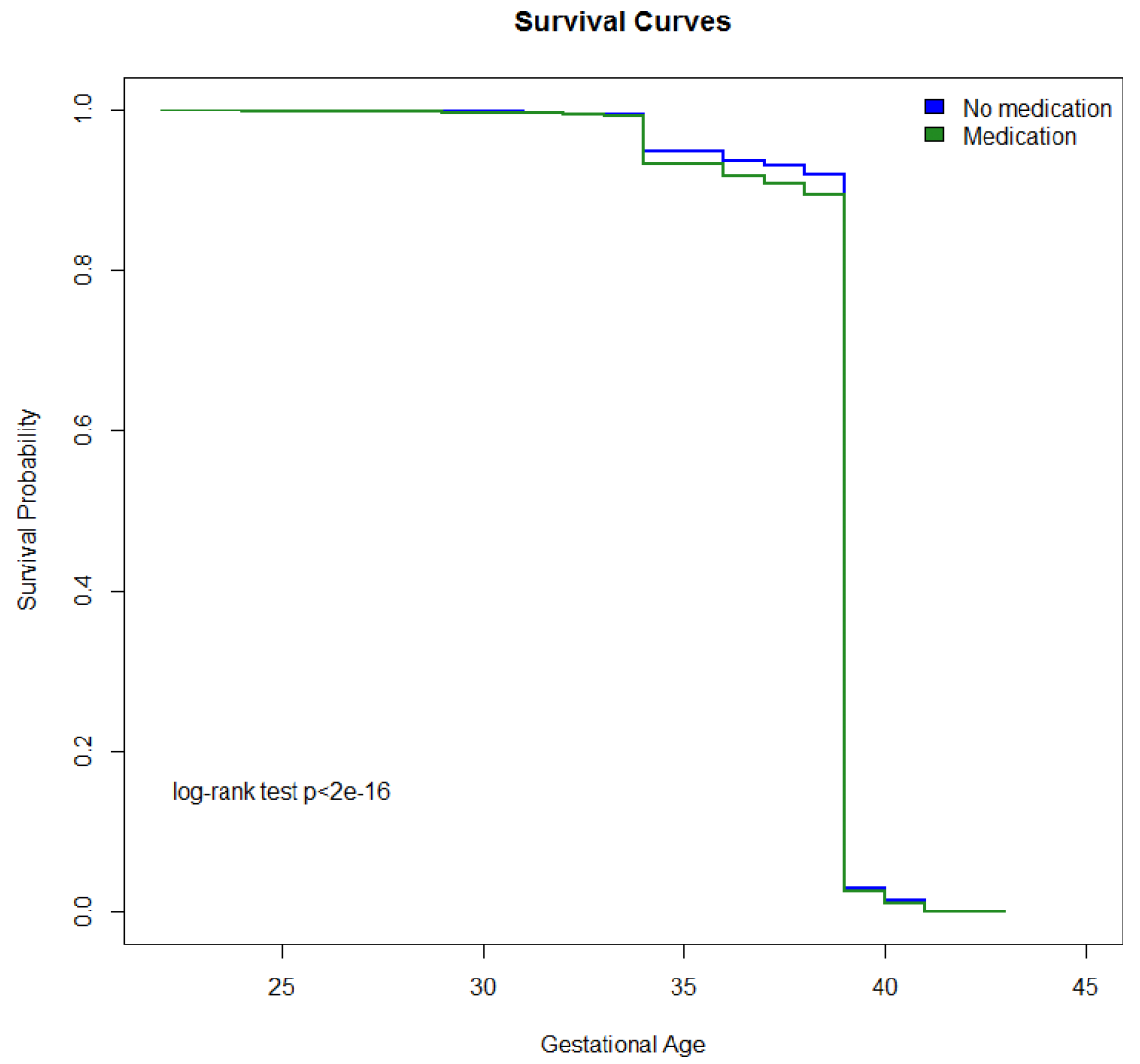


Figure 5. Survival curves for spontaneous preterm birth stratified by Metronidazole and/or Clindamycin treatment for 30 to 39 gestational weeks. Dashed lines show confidence intervals.

Survival Curves

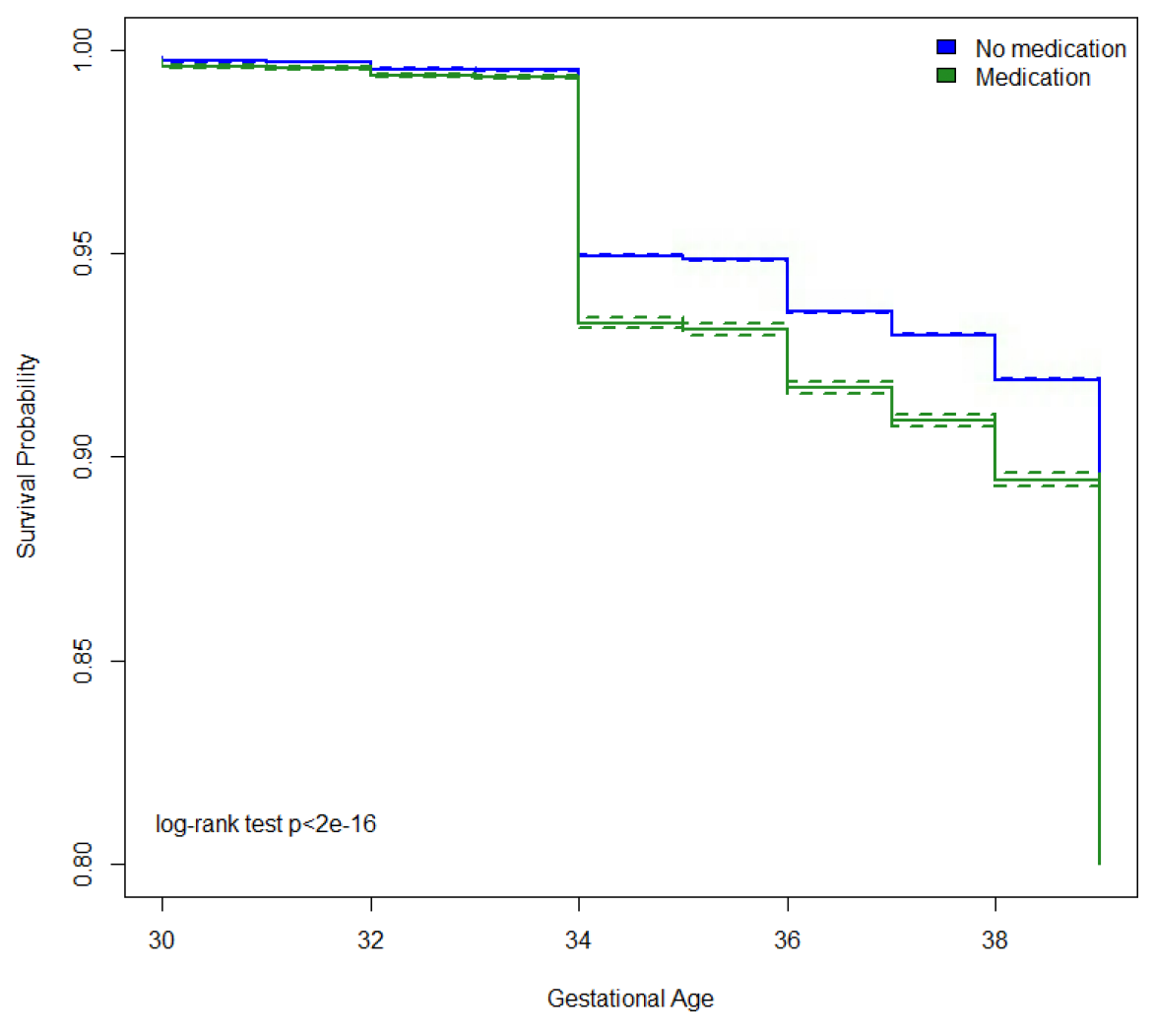

\section{Hosted file}

Figure 1 Pregnancy Cohort.pdf available at https://authorea.com/users/406464/articles/517114treatment-of-persistent-bacterial-vaginosis-and-risk-for-spontaneous-preterm-birth

\section{Hosted file}

Figure 2. Histogram of number of women with BV that filled 1, 2, or 3 and more prescriptions for metron available at https://authorea.com/users/406464/articles/517114-treatment-of-persistentbacterial-vaginosis-and-risk-for-spontaneous-preterm-birth

\section{Hosted file}

Figure 3. Histogram of number of women who filled a prescription per each week of pregnancy.pdf available at https://authorea.com/users/406464/articles/517114-treatment-of-persistentbacterial-vaginosis-and-risk-for-spontaneous-preterm-birth

\section{Hosted file}

Figure 4. Survival curves for spontaneous preterm birth stratified by Metronidazole and:or Clindamycin available at https://authorea.com/users/406464/articles/517114-treatment-of-persistentbacterial-vaginosis-and-risk-for-spontaneous-preterm-birth

\section{Hosted file}


Figure 5. Survival curves for spontaneous preterm birth stratified by Metronidazole and:or Clindamycin available at https://authorea.com/users/406464/articles/517114-treatment-of-persistentbacterial-vaginosis-and-risk-for-spontaneous-preterm-birth

\section{Hosted file}

Table 1. Odds ratios for spontaneous PTB according to treatment for bacterial vaginosis by trimester o available at https://authorea.com/users/406464/articles/517114-treatment-of-persistentbacterial-vaginosis-and-risk-for-spontaneous-preterm-birth

\section{Hosted file}

Table 2. Odds ratios for spontaneous preterm births by number of prescriptions during pregnancy.pdf available at https://authorea.com/users/406464/articles/517114-treatment-of-persistentbacterial-vaginosis-and-risk-for-spontaneous-preterm-birth 